\title{
EL LÉXICO CROMÁTICO Y LA IDEOLOGÍA MAYA
}

\author{
SANJA SAVkIC \\ Doctorado en Historia del Arte, \\ Facultad de Filosofía y Letras, \\ Universidad Nacional Autónoma de México
}

\begin{abstract}
Resumen: Para acercarse al campo semántico de los colores, de primera importancia son diversos diccionarios de las lenguas mayances. El objetivo es hacer una descripción preliminar del vocabulario cromático, compuesto de cinco términos referidos a colores básicos que en el maya yucateco se llaman sak, ek', chak, k'an y ya'ax y significan "blanco", "negro", "rojo", "amarillo" y "verde-azul", respectivamente. El mismo vocabulario remite a determinadas categorías extralinguísticas que la lengua es capaz de acumular, reflejar y comunicar. Éstas dependen de una cultura particular, por lo que el estudio se amplía con las informaciones de diferentes fuentes escritas redactadas durante la época colonial. La intención es detectar los múltiples usos que se hacían en todos los ámbitos de la vida de los colores, lo cual a su vez permite considerar estos últimos como conceptualizaciones articuladas en pares de oposición o agrupamientos tripartitos.
\end{abstract}

Palabras clave: campo semántico cromático, léxico cromático, términos de los colores básicos, color en las fuentes escritas, color, ideología.

AвSTRACT: To gain greater insight into the color semantic field, it is important to consult various Mayance language dictionaries, in order to assemble a preliminary chromatic vocabulary, composed of five basic color terms which in Yucatec Maya are sak, ek', chak, k'an and $y a$ 'ax, or "white", "black", "red", "yellow" and "green-blue", respectively. The very vocabulary addresses certain extra-linguistic categories that language is capable of accumulating, reflecting, and communicating. These categories depend on a particular culture; consequently this study is amplified with greater information from different sources written during the colonial period in order to shed light on the many uses that the colors had in the aspects of human life which, at the same time, can be seen in conceptualizations articulated in pairs of opposition or tripartite groupings.

KeYwords: chromatic semantic field, chromatic vocabulary, basic color terms, color in the written sources, color, ideology.

RECEPCIÓN: 31 de agosto del 2010.

ACEPTACIÓN: 3 de noviembre del 2010. 



\title{
EL LÉXICO CROMÁTICO Y LA IDEOLOGÍA MAYA
}

\author{
SANJA SAVkIC \\ Doctorado en Historia del Arte, \\ Facultad de Filosofía y Letras, \\ Universidad Nacional Autónoma de México
}

El tema del presente trabajo gira en torno a la descripción del vocabulario de distintas lenguas del grupo maya que se refiere al campo semántico de los colores, aunado con algunas referencias tomadas de las fuentes escritas. ${ }^{1}$ En primera instancia el corpus se obtuvo a partir de los diccionarios del maya yucateco y aquellos que recopilan palabras del k'iche', kaqchikel y tzutujil. ${ }^{2}$ Los diccionarios más citados son: Diccionario maya Cordemex, Combined Dictionary: Concordance of the Yucatecan Mayan Language y el Calepino de Motul, por un lado. Por el otro, [Thesavrvs Verborü] Vocabulario de la lengua cakchiquel v[el] guatemalteca, nueuamente hecho y recopilado con summo estudio, trauajo y erudición, Primera parte del Tesoro de las lenguas cakchiquel, quiché y zutuhil, en que las dichas lenguas se traducen a la nuestra, española, así como K'iche'-English Dictionary and Guide to Pronunciation of the K'iche'-Maya Alphabet. De modo complementario, el estudio fue enriquecido con la información de otros diccionarios de diversas lenguas mayances, puesto que percibí más similitudes que diferencias al identificar el campo léxico cromático. Esto apunta a que en cuanto a este tema particular, a través de la terminología de las lenguas emparentadas entre sí se percibe una ideología ${ }^{3}$ compartida. Debo advertir que también hay ciertas diferencias, las mismas que se refieren, antes que nada, a la pronunciación y la ortografía (cf. Kaufman y Justeson, 2003) y no tanto, según considero, a los significados y las acepciones abarcadas. No obstante, puesto que el territorio en el cual se hablan los distintos idiomas del grupo maya es vasto y abarca diferentes regiones geográficas y medioambientales o climáticas, los referentes con los que se combinan las palabras de colores pueden variar. Por ejemplo, el blanco para los hablantes del maya yucateco se relaciona con las nubes, mientras que para los pueblos de los altos del área maya se asocia con la nieve y el granizo, experiencia que está ausente en la vida de los mayas peninsulares. Al mismo tiempo, recurro a distintas fuentes escritas tanto

\footnotetext{
${ }^{1}$ La presente investigación toma como base algunas ideas de mi tesis de maestría titulada "El color en el Códice de Dresde según la información de las fuentes escritas y de las linguiísticas” (2007).

${ }^{2}$ Respetaré la ortografía original de las lenguas mayas así como aparece en distintos libros cuando los cito. En el resto de los casos, usaré la ortografía modernizada.

${ }^{3}$ El término ideología lo uso para designar un conjunto de ideas, conceptos, prácticas, actitudes y valores de una sociedad.
} 
por los españoles como por los indígenas, redactadas en diferentes momentos de la época colonial, puesto que "el pensamiento simbólico establece relaciones entre elementos de la realidad y conceptos" (Dehouve, 2003: 64) así como para subrayar la relevancia que han tenido los colores para los mayas.

¿Por qué es válido el estudio de las palabras? De acuerdo con Gabriel Bourdin, no se trata de simples nombres superpuestos a las "cosas" o "vivencias" naturales. El significado léxico (al igual que el significado gramatical) es un conjunto de perspectivas específicas que a su modo "construyen" percepciones y evaluaciones de la realidad, y lo hacen según la modalidad y el estilo de una determinada lengua y de una cultura (Bourdin Rivero, 2008: 22-23). Para el mismo autor el estudio de las palabras es un paso necesario que nos permite ir, si así lo deseamos, más allá de las palabras (23). Los nombres de los colores representan un conjunto ordenado que se relaciona con otros campos semánticos para producir nuevos significados (a través de las formas compuestas como sak be, sak ool, en el maya yucateco); o bien lo hacen por su propia polisemia (sak significa blanco y falso, entre otras acepciones). El mismo estudio también permitiría sugerir ciertos criterios para la posible clasificación de dichos vocablos, como se verá a continuación.

Los propósitos de esta investigación son determinar cuál es el campo específico de la nomenclatura cromática y qué categorías extralinguísticas es capaz de acumular, reflejar y comunicar, las cuales normalmente dependen de una cultura particular. Igualmente, las fuentes escritas son de gran ayuda para detectar los múltiples usos que se hacían de los colores en todos los ámbitos de la vida, mismos que a su vez permiten hablar de las conceptualizaciones o categorías cromáticas articuladas en pares de oposición o agrupamientos tripartitos.

Detecté dos grupos de palabras de colores: los básicos y los derivados. El término color básico lo uso según lo emplean Brent Berlin y Paul Kay en su libro publicado por primera vez en el año 1969 titulado Basic Color Terms: Their Universality and Evolution, basado en el estudio de 98 diferentes lenguas del mundo, ${ }^{4}$ mientras que los colores derivados, por consiguiente, serían todo lo que aquellos no son. Con más precisión, se trata de las palabras compuestas por un término de los colores básicos y otra(s) palabra(s) para expresar colores llamados secundarios en la cultura occidental o sus diferentes matices. Este estudio polémico representa un buen punto de partida para muchas cuestiones que se refieren al tema del color.

En la terminología de Berlin y Kay los nombres de los colores básicos ${ }^{5}$ son aquellos que deben cumplir con las siguientes características: 1) deben ser mo-

\footnotetext{
${ }^{4}$ Veinte idiomas de familias no relacionadas fueron investigados por los autores como parte de un trabajo de campo, mientras que el resto de la información proviene de diferentes fuentes bibliográficas. Cf. Berlin y Kay, 1991.

${ }^{5}$ Para hablar de colores básicos, los autores emplean el término punto focal, que sería algo como, por ejemplo, "el rojo más representativo" de todo el espectro de colores presentados en la tabla de Munsell. Plantean la existencia de universales semánticos en el vocabulario del color y el desarrollo
} 
nolexémicos (el significado de la palabra no es predecible al analizar sus partes); 2) su significado no se incluye en aquel de otro término; 3) su aplicación no debe estar restringida a una limitada clase de objetos; 4) debe ser sicológicamente notable para los hablantes (Berlin y Kay, 1991: 6). Se nota que estos criterios cubren una amplia gama de las prácticas humanas que abarcan el dominio de la lengua (morfología, sintaxis y semántica en este caso particular), los usos de los objetos que dependen de diferentes necesidades y costumbres de las personas, así como la percepción (una condición biológica, física y sicológica).

Varias lenguas indígenas que fueron investigadas en México —el ixcateco, mazateco, popoluca de la sierra, tarasco, tzeltal y tzotzil—, según estos autores pertenecen al estado IV (de siete en total) de evolución sociolinguiística, lo que significa que reconocen cinco términos de colores básicos (de once en total): blanco, negro, rojo, verde y amarillo, en este orden específico. Igualmente, Berlin y Kay indican que en el caso de la mayoría de las lenguas autóctonas de México y América Central el verde y el azul se designan con una sola palabra.

En el caso de las lenguas del grupo maya la palabra para estos dos colores es $y a$ 'ax o $y a^{\prime} x$ (en el maya yucateco), o räx o rax (en el k'iche', kaqchikel y tzutujil). La gran mayoría de los informantes tzeltales de Berlin y Kay ubicaron este color entre los verdes en la tabla de Munsell. Por otro lado, en todos los diccionarios mayas revisados ya'ax se utiliza tanto para el verde como para el azul, y se habla del verdor de la naturaleza y la profundidad de las aguas, así como del azul del cielo, como se dice en el Diccionario maya Cordemex. De todas formas, es imprescindible subrayar que el hecho de usar una palabra para dos colores del espectro no significa la incapacidad biológica ni física de sus hablantes, sino que se trata de un fenómeno cultural, ya que los pueblos distinguen y nombran aquellos colores que tienen importancia para ellos. Por lo tanto, a nivel de la percepción se trata de dos colores, pero a nivel semántico de uno solo. Tampoco hay que olvidar el factor medio ambiental, ya que la naturaleza del subtrópico se manifiesta con una gran riqueza de flora y fauna ricamente coloreadas.

Igualmente, en la mitología y las creencias autóctonas tal y como se nos presenta en el Popol Vuh escrito en la lengua k’iche', se encuentra la narración más completa del origen del universo. ${ }^{6} \mathrm{Al}$ inicio de la historia se advierte cómo los dioses creadores —que constituían una pareja masculino-femenina_-, ${ }^{7}$ empren-

histórico de los mismos en la lengua. Sus dos hipótesis centrales son: 1) la categorización de los colores no es una casualidad y el ámbito semántico de los términos básicos del color es igual para todas las lenguas, y 2) existe una secuencia fija de siete estados evolutivos por los cuales pasa cada lengua, así como el incremento de su vocabulario básico en un orden fijo (son once categorías básicas de color: blanco, negro, rojo, verde/amarillo, azul, café, morado, rosa, anaranjado y gris). Todas las lenguas del mundo pueden ser ubicadas en uno de los siete estados, lo cual indica el grado de desarrollo tecnológico y cultural de un grupo humano (Berlin y Kay, 1941).

${ }^{6}$ La historia es corroborada en el Memorial de Sololá de los cakchiqueles y en los Libros de Chilam Balam provenientes de Yucatán.

${ }^{7} \mathrm{O}$ un dios andrógino, según Mercedes de la Garza 2002: 57. 
dieron la generación del mundo estando todavía sobre el agua primigenia y en el momento del caos, con la finalidad de crear al hombre para ser venerados y alimentados. Algunos nombres de estas deidades son metáforas asociadas con su función, de las cuales unas se forman con los adjetivos de colores u otras palabras que fungen como referentes importantes para un determinado color.

Abordando todavía el complejo verde-azul, me detendré en el segundo término de la quinta pareja que se llama Q'ukumatz, ${ }^{8}$ versión quiché del K'ukulk'an yucateco y del Quetzalcóatl tolteca. Q'uk $k^{9}$ significa "plumas verdes", forma que se utiliza también para denominar al ave quetzal; también es una forma de expresar que algo es de alto valor o precioso, mientras que kumatz ${ }^{10}$ es "serpiente". La séptima pareja divina está conformada por Ah Raxá Lac y Ah Raxá Tzel (al parecer deidades masculinas como lo revela la palabra $a h$, "el", usada para designar a los masculinos), que se traducen como "Señor del Verde Plato" y "Señor de la Jícara Azul", respectivamente. Aunque ambos utilizan el término raxá, es sabido que el mismo podía ser utilizado tanto para el verde como para el azul. La propuesta de traducir el segundo término como azul surge de la traducción directa realizada por el padre Ximénez en la columna derecha del manuscrito original.

Raxón es una forma de denominar a un ave de plumas de color azul celeste. En el diccionario K'iche'-English de Allan Christenson la palabra q'uq' designa a "quetzal bird". Recinos comenta que guc o q'uc en k'iche', y k'uk' en el maya yucateco, es el ave que hoy se llama quetzal (Pharomacrus moccino). El mismo nombre se usa para las plumas verdes de la cola de esta ave, llamadas quetzalli en náhuatl. Raxón o raxom (Cotinga amablis) es otra ave de plumaje azul celeste. ${ }^{11}$ De tal forma que Ah Raxá Lac y Ah Raxá Tzel corresponderían en ese orden a los señores de la tierra ("verde plato") y del cielo ("jícara azul"). En el mismo diccionario se señalan dos frases adjetivales: una, räx räj u wo ri kaj que significa "blue like the sky" y otra, räx räj u wo ri q'ayes, "green like the grass".

Recordemos que antes de la creación del mundo, el cielo y la tierra estaban unidos y que las deidades se encontraban debajo de las plumas verdes y azules, lo cual probablemente apunta a un solo término usado para designar los colores verde y azul en todos los idiomas mayances. El primero normalmente se asocia con lo terrenal y el segundo con lo celeste, ámbitos que en el momento primordial eran un todo en forma acuática.

Solamente había inmovilidad y silencio en la oscuridad, en la noche. Sólo el Creador, el Formador, Tepeu, Gucumatz, los Progenitores, estaban en el Agua rodeados de claridad. Estaban ocultos bajo plumas verdes y azules, por eso se les llama Gucumatz.

\footnotetext{
${ }^{8}$ Gucumatz según la ortografía que emplea Adrián Recinos en su edición de Popol Vuh, las antiguas historias del Quiché.

${ }^{9}$ Guc (Popol Vuh, 2003).

${ }^{10}$ Cumatz (Popol Vuh, 2003).

${ }^{11}$ Las plumas de estas dos aves tropicales, que abundan especialmente en la región de Verapaz, eran usadas en los adornos ceremoniales de los reyes y señores principales desde los tiempos más antiguos de los mayas (Popol Vuh, 2003: 166).
} 
De grandes sabios, de grandes pensadores es su naturaleza. De esta manera existía el cielo y también el Corazón del Cielo, que éste es el nombre de Dios. Así contaban (Popol Vuh, 2003: 23).

Tepeu y Q'ukumatz aparecen aquí como divinidades progenitoras que, rodeadas de claridad en medio de la omnipresente oscuridad, están ocultos bajo plumas verdes y azules; nuevamente aparecen los términos para los colores: pa guc ("verde") y pa raxón ("azul”). De hecho, vemos que es posible el uso de dos palabras distintas para denotar estos dos colores del espectro visual. En el diccionario del maya yucateco presentado por Bolles, este autor dice que yax es "green, blue", pero agrega también lo siguiente: "While there existed the word chhoh for blue, which is also applied to the indigo bush, today yax is the only word which is applied to both colors green and blue". ${ }^{12}$ No obstante las posibilidades de recurrir a distintas palabras para designar dos colores del espectro visual, siempre hay que tener en mente que para los mayas estos dos colores forman un complejo nombrado a través de un solo término.

Al mismo tiempo, hay que tomar en cuenta la importancia del maíz que tiene cuatro variedades considerando el color del grano denominado imix: blanco, amarillo, rojo y negro. Por otro lado, existe otra palabra cuando se habla de la planta considerada junto con el follaje (nal), lo que remite al color verde. Me parece pertinente mencionar el mito del descubrimiento del maíz con referencia a sus diferentes colores, que relata J. Eric. S. Thompson en su libro Historia y religión de los mayas. El autor señala que el mismo mito del descubrimiento del maíz debajo de una roca era compartido por los pueblos del Altiplano Central de México y los diversos grupos mayas. Escojo como ejemplar la versión de los mopanes de San Antonio, Belice, que al mismo tiempo es la más completa y es compartida por los kekchís y pokomchís. Como el maíz estaba oculto bajo una gran peña, sólo las hormigas tenían el acceso a él. Muchos animales se quisieron aprovechar y el hombre también. Éste último pidió la ayuda de los dioses del trueno, los Mames, para que enviaran la lluvia; de los cuatro, el principal y el más grande era Yaluk, quien finalmente logró deshacer la roca. Al principio todo el maíz había sido de color blanco, pero el rayo de este dios quemó algunos granos y se volvieron rojos, y ahumó otros que se hicieron amarillos, por lo que ahora existen mazorcas de diferentes colores. Thompson indica que al informante sin duda se le olvidó mencionar al maíz negro, carbonizado por el rayo (Thompson, 2004: 417-425).

En las lenguas mayas los nombres de cinco colores básicos — sak, ek', chak, k'an, $y a a^{\prime 13}$ ("blanco", “negro", “rojo”, "negro”, “amarillo”, "verde-azul”)— son

\footnotetext{
${ }^{12}$ Hay que tomar en cuenta que en el Diccionario maya Cordemex la palabra para los colores verde y azul es $y a$ 'ax o $y a^{\prime} x$, mientras que yax tiene otro significado, el de "primer(o)". Por otro lado, la grafía correcta para el segundo término referido por Bolles es ch'oh en el Cordemex y significa "añil", "yerba de añil, y la planta que lo da". Finalmente, hay que notar que la ortografía que usa Bolles depende de las fuentes que cita.

${ }^{13}$ En adelante usaré como términos tipo aquellos del Diccionario maya Cordemex para facilitar la lectura. Por otro lado, me referiré a la grafía original al citar directamente diversas fuentes.
} 
clasificadores importantes. Aproximadamente una tercera parte de los términos vegetales y animales están ligados al color. Además, con los colores se pueden designar diferentes enfermedades, expresar estados emocionales, así como indicar diferentes tipos de tierra/suelo y sus propiedades y usos. También los nombres de diferentes metales se construyen con los términos de colores, así como múltiples topónimos y patronímicos. Al mismo tiempo, el espacio y el tiempo se organizan mediante los colores: el espacio — con el sentido de plano cósmico- a través de los puntos cardinales y su centro (este: rojo; norte: blanco; oeste: negro; sur: amarillo; centro: $y a^{\prime} a x$ ). Por su parte, el tiempo se vincula por medio de los días, meses y años a diferentes colores y deidades que los rigen, lo cual posee mucha importancia en distintos rituales. En la cosmovisión maya el concepto del tiempo no se separa del concepto del espacio; ambos a su vez van aunados a otros diversos elementos (deidades, plantas y animales).

En el sexto capítulo sobre los calendarios, ritos y escritura, fray Diego de Landa nos informa sobre estas cuestiones, donde la frase "letra dominical" se refiere a los cuatro días del tzolk'in (ciclo de 260 días) que coincidían con el primer día del primer mes del haab (ciclo de 365 días), llamados cargadores del año y que tenían gran importancia ritual, los mismos que en tiempos de la conquista eran K’an, Muluk, Ix y Kawak:

La primera, pues, de las letras dominicales es Kan. El año que esta letra servía era el agüero del Bacab que por otros nombres llaman Hobnil, Kanalbacab, Kanpauahtun, Kanxibchac. A éste señalaban a la parte de medio día. La segunda letra es Muluc; señalábanla al oriente y su año era agüero el Bacab que llaman Canzicnal, Chacalbacab, Chacpauahtun, Chacxibchac. La tercera letra es Ix. Su año era agüero el Bacab que llaman Zaczini, Zacalbacab, Zacpauahtun, Zacxibchac y señalábanle a la parte del norte. La cuarta letra es Cauac: su año era agüero el Bacab que llaman Hozanek, Ekelbacab, Ekpauahtun, Ekxibchac; a ésta señalaban a la parte del poniente (Landa, 1985: 104-105).

En esta cita claramente se ve que los colores forman parte de los nombres de las deidades mencionadas y que al mismo tiempo se relacionan con un punto cardinal y un día específico. Al mismo tiempo, determinados meses del calendario solar tienen nombres formados con los términos de los colores, sobre todo cuando se toman en cuenta aquellos que se usaban durante en el periodo Clásico: el primer mes es K'anjalaw o K'anjalab (Pop), ${ }^{14}$ el tercero es Chakat (Sip), el séptimo Yaxk'in, ${ }^{15}$ el noveno Ik'sihom (Ch'en), el décimo Yaxsihom (Yax), el onceavo es Saksihom (Zac), el doceavo Chaksihom (Ceh), el mes catorce es Uniw (Kankin) y el diecisiete es K'anasiiy (Kayab). Es importante mencionar que los

\footnotetext{
${ }^{14}$ La primera palabra se refiere al nombre usado en el periodo Clásico, mientras que la segunda (aquella entre paréntesis) es del maya yucateco del siglo xvi (Kettunen y Helmke, 2008: 46).

${ }^{15}$ La traducción correcta de este término es "primer sol" y no "sol verde/azul", ya que la palabra yax — con la vocal corta y sin salto glotal— en el maya yucateco significa "primer(o)" y no designa al color.
} 
mismos glifos se usan para escribir las palabras "verde, azul" y "primer(o)", ya' $a x$ y yax, respectivamente, por un lado, así como para "amarillo" y "precioso", k'an, por el otro (Kettunen y Helmke, 2008: 46).

Prácticamente, los colores son elementos omnipresentes y abarcan diferentes aspectos tanto de las prácticas religiosas como de la vida cotidiana. Por ejemplo, por la Relación de las cosas de Yucatán de Landa sabemos que las personas se pintaban el cuerpo completo de negro dos o tres meses antes de la fiesta del Año Nuevo en señal de penitencia. Las ceremonias de mayor importancia eran las que se hacían en los últimos cinco días del haab llamados Wayeb', cuando se preparaba la llegada del nuevo ciclo y se renovaban todas las cosas del servicio, se limpiaban las calles, se adornaban los templos y se bañaban los participantes para quitarse el tizne y expulsar así la suciedad acumulada en el transcurso del año pasado (Landa, 1985: 130-132).

Por otra parte, en cuanto a las costumbres de guerra, en diversas fuentes escritas se encuentran numerosas evidencias sobre el arreglo personal y su relación con la pintura corporal. La guerra, como factor importante de la sociedad maya, tenía sus propias reglas y ceremonias. Así, la fiesta principal del mes Pax era la de los guerreros. En el Diccionario maya Cordemex se halla la frase chak pax que significa "tambor de guerra", donde pax se refiere al "tambor" y chak por su parte designa a "rojo, grande, fuerte, aguacero" — entre otros significados de los que se hablará más adelante-, pero que en este caso particular se refiere a la guerra. ¿Cuál sería la relación entre esta última, el tambor y el color rojo?

La información sobre la costumbre de los guerreros de pintar sus cuerpos se encuentra en las Relaciones histórico-geográficas de la gobernación de Yucatán, donde se indica que se pintaban de negro y rojo, como en la "Relación de la Ciudad de Mérida": "Y por mostrar ferocidad y parecer más fieros y valientes se embijaban de negro y con almagre los ojos y narices y todo el rostro, cuerpo y orejas" (Garza, I, 1983: 67). En la "Relación de Tabi y Chunhuhub" se dice que los hombres, mayormente desnudos y embijados de negro en señal de tristeza o ira, salían a las batallas con sus capitanes y mandones (Garza, I, 1983: 165). Vemos que al hablar de la pintura del cuerpo, los cronistas refieren sus valencias sicológicas y las interpretan de diversas maneras. Para ellos los colores negro y rojo usados en estas circunstancias especiales se asocian con la ferocidad y la valentía, pero también con la tristeza y la ira. No está claro si estas conclusiones son sus apreciaciones personales o tienen fundamento en las creencias de los mismos indígenas.

Para no detenerme más en la importancia del uso de los colores precisos en distintos rituales como, por ejemplo, el azul para el sacrificio, sólo agregaré que para la ocasión del casamiento, los jóvenes se quitaban el tizne (es decir, el color negro apropiado para el ayuno previo a la boda), para posteriormente pintarse de rojo (Landa, 1985: 96). Landa señala que, cuando no era el tiempo de ayuno, las mujeres indígenas, igual que sus maridos (los que tenían la posibilidad), solían untarse con cierto ungüento colorado, es decir, rojo: "Que usaban pintarse 
de colorado el rostro y el cuerpo y les parecía muy mal, pero teníanlo por gran gala" (Landa, 1985: 74). Probablemente el rojo también se utilizaba para decorar partes del cuerpo y no tenía un significado ritual, sino social de lucimiento o de exaltación de la belleza, un aspecto meramente de decoración en la vida cotidiana.

Por último, en el Calepino de Motul se encuentra la frase hobon ool, que significa "hábil, ingenioso, inventivo y sabio". Aquí hobon es palabra genérica tanto para "color o matiz" como material para pintar, así como para la "cosa pintada o labrada de colores", y ool es "corazón (anímico)". Lo que importa es que el hecho de tener color(es) es una característica de alto valor.

\section{El léxico cromático}

¿Cómo se constituyen los significados con los nombres de los colores y cuál sería la manera más directa de conocerlos? Partiendo de la idea de que el lenguaje natural es muy plástico, al unir las palabras que a veces designan cosas y aspectos comunes, se producen otros significados: los figurativos. También, al tener una palabra varios significados, su comprensión y traducción se dificultan, pero al mismo tiempo se enriquecen las posibilidades de sus interpretaciones.

Considero que uno de los métodos para responder estas preguntas es aislar las palabras de los colores, conocer todas las acepciones que se construyen a partir del nombre de un color y de un referente externo mediante distintos tipos de asociaciones, que finalmente llevan a la conceptualización. Posteriormente, al comparar entre sí los diferentes colores con todos los significados generados, se correlacionan y oponen (al considerar sus semejanzas y diferencias), formando así pares o grupos de categorías que producen sentido para los hablantes de una determinada lengua.

\section{Colores básicos}

Aquí presentaré aquellos significados de los términos básicos de colores y las formas compuestas con estos términos que, según mis criterios personales, son los más representativos.

Sak

En el Diccionario maya Cordemex se señala que la palabra sak en primer lugar se refiere a "cosa blanca" y luego también a lo "claro, limpio, suave y delicado", al "alba" o a la "mañana, cuando amanece o entre dos luces". Así, sak t'in kab es la "luna muy clara" y sak muyal son las "nubes claras o ralas", mientras que sak naen yahal kab significa "de día, cuando quiere salir el sol, que ya blanquean las casas". 
Sak hop'e'n es "cosa blanca con lustre que reluce, como plata”, "cosa blanca y resplandeciente". Sak-hii' significa "lavar y limpiar el maíz para hacer pan que quede blanco". Sak ik' es "viento fresco suave y delicado, viene de donde sale el sol”. Sak tso'tsil designa a las “canas que se hacen por ser ya viejos”. Sak tak'in es "dinero, moneda de plata”. La palabra sas también significa "blanco". A parte de este significado, designa la "luz, claridad, claro, no obscuro, resplandor de día, iluminación o aclaración", y lo "manifiesto, iluminado o diáfano". Sas ol es "bueno, de buenas, de claras y limpias entrañas" y como sak ool se refiere a los "pulmones”. Sasak tok' es "pedernal blanco o bueno".

En composición con algunas palabras denota cosa artificial, como sak na', "madre artificial o no auténtica, madrastra", y sak mehen, "hijastro", o aquella cosa hecha por la mano humana, como sak be que es "calzada”. Además, también en composición con otras palabras, disminuye la intensidad o denota cierta imperfección: sak pakat, "mirar con disimulación, fingir que no ve”. Sakan significa "no cocido" y sakan wah se refiere al "pan hecho de masa, mal cocido". La frase sak wayak' se refiere a los "sueños sucios, feos y deshonestos, y soñar así” o "soñar cosas feas”. Sakpil'en es "pálido”, sakpak'e'n significa "el que tiene el rostro blanquecino y demudado por alguna enfermedad, perturbación o miedo”, mientras que sakpak'e'n u wich es “desfigurado, blanca cosa o blanquecina de color muerto".

Adicionalmente, en el Thesaurus de fray Thomás de Coto se dice que çak es "blanca cosa, color, clara cosa, claro, hermosa cosa, luz, medio o parte, medir, muy". Çak aqual: ru es "nacer", mientras que çak bakon, "carámbano, helada, nevar, niebla”. Çak r'ih son las "canas”, mientras que çak puluvan significa "espuma”. Çak cahcoh/cahcohil se refiere a "blanca cosa" y a "descolorida ponerse". Cubre también el espectro del amarillo: çak bakaboh/bakabohil/bake/bakeic.

Además, en el Diccionario de fray Francisco Ximénez el morfema zac también denota "suavidad, agudeza". Zacamac es la "paz". Zahac-q es "diligente, mañoso, sabio". Zacacic es "cosa que está medio quebrada”. Zacbaque, ti significa "mudarse de miedo o espanto", mientras que zac cahe, ti es "ponerse descolorido". Zac caracoh se refiere a la "cosa muy fría". En el mismo diccionario se usa otra grafía, zaq. Zaquil designa "claridad, blancura; mentira", zaquichuh denota al "loco, endemoniado", zaquetal es "da a entender que con poco se ensoberbece, como con dinero". Finalmente, zaquibachonel es "el adivino" y zaquiuachin, ti significa "adivinar por sueños, hacer hechizos".

Denota la intensidad en las palabras compuestas como en çacach tan, "hablar mucho o demasiado" y çac bul ik, "tempestad grande de viento". ${ }^{16}$

En el Cordemex también aparece otra traducción para la palabra compuesta con sak-sakal, que significa "tela en el telar, tela en general, tejido, tejer".

${ }^{16}$ Los dos ejemplos son de Calepino de Motul, citado por Alfredo Barrera Vásquez (1981: 48). 
En el Cordemex ek' es “cosa negra, ennegrecer, oscurecer, hacer una cosa negra, ensombrecer”. Ek'tablahoy ti' yotoch k'u' ma'mak yani' significa “sola, obscura, lóbrega y tenebrosa está la iglesia, no hay nadie en ella”. Ek' a ich to'on designa "huraño, bravo, desamorado, extraño, indómito, montaraz, intratable, zahareño, que no se deja tocar ni se llega a nadie". Ek' be es "senda o camino agro o estrecho y ciego o angosto", "camino áspero, poco usado o no usado". Ek’bis (o ek'bise'n) es "cosa oscura, oscuridad" o "cosa obscura y tenebrosa o lóbrega". Ek'bise'nil significa "obscuridad y tinieblas así, la oscuridad de la noche, tinieblas, tenebrosidad”. Ek'bit'e'n designa "cosa negra o sucia como ropa de cocinero percudida o cera así negra”. Ek'bon es "tinta negra”. Ek' bukil es "luto”. Ek' buts'e’n significa "cosa negra como humo o ahumado". Ek'mal es "cocer demasiado". Ek' maychahal significa "ponerse o quedar ciego". Ek' taw es "plomo (metal)". Ek' Chuwah es "patrón de los comerciantes y de los sembradores de cacao ['alacránnegro']".

En el Thesaurus del padre Coto se acotan también los siguientes significados: “matar, relajar" para 3ek r'ey. 3ek ru vach es "enturbiar". 3ekal(: xa) se refiere a "blandamente, con tiento, paso, poco a poco; mansa persona; negra cosa”. 3ekal hab es "tiempo lluvioso". 3ekariçah: tin se refiere a "color [negro], ennegrecer, ensuciar, negra cosa”, 3ekariçah ru vach: tin es "afear y estragar”. 3ekum vachil significa "desvanecerse" y 3ekumal chi tzil es "secreta cosa".

En el Diccionario de Bolles se refiere a "twilight, dusk" ("crepúsculo, penumbra, atardecer, al anochecer"). El mismo autor indica que el sinónimo de $e k$ ' es box en el maya yucateco y aparte de referirse al "color negro y la oscuridad", también designa a la "concha, piel, cáscara, corteza (de árbol)" y "la cueva con agua”. Además, denota lo "malo y perverso".

Chak

Según el Cordemex, la palabra chak tiene varios significados. Puede ser apellido y como tal significar "rojo, grande, fuerte, aguacero". También significa "cosa colorada”, mientras chak p'oxe'n es "cosa muy colorada, bermeja o encendida”. En composición con otras palabras significa "muy, mucho o muy bien”, y al mismo tiempo "gran cosa o negocio" y también "cosa grave y de mucha importancia”. Chak también es "cocer en agua o en otro licor" y "sazonar". Chakaw se refiere a la "cosa caliente o calurosa que tiene calor y la calentura y calor". La palabra chak en composición con otras designa los estados de ánimo como la ira o la cólera, enojarse: chakxike'nhil u wich es "encenderse [su rostro] por ira o cólera", mientras que chakxike'n $u$ wich significa "desnudarse de vergüienza, encenderse el color de verguienza, robustez". Chachak-kunah es "envejecer, poner o teñir de rojo, enrojecer". Chakaw olal denota "cólera, enojo encendido y tenerle y encolerizarse, ira y enojo rabioso". Chakaw es "cosa caliente o calurosa que tiene calor 
y calentura o calor". Chakan son "plumas de la cola de guacamaya". Chakawhal ol, chakawhal lu'um significa "cansarse la tierra y esquilmarse y encolerizarse el hombre". Chakaw k'ab se refiere al "que tiene mala mano para algo (para plantar [rasgo cultural que se refiere a la "cualidad" fría o caliente de las cosas])", como se dice en el Cordemex. Chak sits'il es "avaricia y codicia". Chak / chachak maskab se refiere al "cobre".

En el Thesaurus cak se refiere a "áspera cosa, colérico, color, cruel persona, impaciente". Cakariçah: tin es "color, provocar", mientras que cako un vach chire: ti se refiere a la "envidia”. Cak çiquih es "hechizo". Cak chachoh denota "seca cosa", cak chuluchuh: ti es relumbrar. Cak pupuh v'ih es "calentura”, cak vach: ah "malicioso", cak vachibal rijl "envidia" y cak vachich: tin "odio". Cak también es intensificador del significado de otras palabras y modificador de nombres. Cak hay un qohlem: quere significa "rico". Cak i3 designa "aire, huracán”, cak rax se refiere al "color”, mientras que cakix es "ciruela, guacamaya, papagayo".

Fray Francisco Ximénez indica los siguientes significados para la palabra 3a3: "el fuego y cosa colorada", "la luciérnaga”. 3a3ab es "bellaco y malo", "respiración de fuego". 3a3ah, tin significa "herrar animal o esclavo con fuego", "tener envidia, o dolor de ardor"; "afligirse, tener comezón, y arder el sol”. 3a3al es "grandeza, majestad; calentura", mientras que 3a3bachih, tin designa "tener celos, levantar falso testimonio, o sospechar en mala parte". 3a3rail significa "con dificultad". 3a3tihax-q se refiere al "lujurioso", mientras que 3a3zutcum a "remolino de viento, gran enojo".

K'an

En el Diccionario maya Cordemex, k'an se refiere a "cosa amarilla, color amarillo". K’ankobe’n designa "amarillo blanquizo como maíz de filo medio podrido". La palabra k'ank'anil significa "lo amarillo o que tiene este color", mientras k'antop'e'n significa "florido campo, campo amarillo; amarillo como el de las flores de este color". K'an ts'ile'n es "amarillo, como la mazorca de maíz que ha sazonado", mientras k'ants'ile'nil es "lo amarillo y el estado de madurez de la mazorca de maíz por estar al secarse”. También, k’an es "fruta madura o sazonada”, mientras k'anil es "sazón de las frutas, aunque no estén maduras, por estar las más tomando el color amarillo". K'an mech'en es "descolorido”, mientras que k'an pachen es "descolorido de miedo o por alguna enfermedad". K'an tak'in es "oro o moneda de oro". K'an también significa "piedra preciosa; piedras de valor; adorno; conchas amarillas".

En el Thesaurus se indica que 3an significa "abohetado, amarilla cosa, color [amarillo], rubia cosa". Además, 3an: qa çak se refiere a "fruta; madurar, madurarse”. 3an: qo chic ru es "preñada”, mientras que 3an chic es "blanquear; mazorca”. 3an yaal: nu designa "sacrificar hombres; sudar". 3ana vinak significa "atestiguar, negar, testigo". 3ana puvak es "oro", mientras que 3anal: nima es "estado de gloria”. 3anal raxal se refiere a "amarilla cosa, próspera cosa” y 3anal raxal: nima es 
"gloria, paraíso". Hay que notar que en estas dos últimas frases los elementos constitutivos son 3an y rax.

Ya'ax

Para los mayas los colores verde y azul se hallan en la misma categoría cromática, así que ellos no hacen una distinción explícita en cuanto al término que los designa y se los denomina conjuntamente ya'ax. Se trata de un aspecto puramente conceptual del esquema cromático construido desde su cultura, según el cual estos dos colores no pueden pertenecer a diferentes categorías (Vargas Melgarejo, 1998: 88).

En el Cordemex se indica que ya'ax significa "verde", mientras que la palabra ya'ax k'uk' significa "verde oscuro que tira a azul". Con otras palabras forma frases que designan "cosa verde, álamo verde, verde fino, fruta que cae del árbol verde y sin sazón, verdura, lo verde”. Ya'aya'ax k'ante; yaya'ax u xe se refiere a "cólera verde, entre azul y verde, cosa verde no madura, verduzco". Ya'xkab significa "azul, cosa clara o azul aturquesado", igual que ya'ya'ax, ya'xtop'e'n y ch'ooh. Esta última palabra también se refiere a la "planta de añil, índigo”. (Ah) ya'x bak' es "descolorido; cosa abuhetada, hinchada”, mientras que ya'xbak' ya'axbak' se refiere al "hombre enfermizo o que anda siempre enfermo". Ya'xhal significa "ponerse verde o reverdecer". Ya'x hoh denota "milpa de sólo frijoles en tiempo de aguas, que aún no está quemada ni sembrada pero está arrancada la yerba y cortados los árboles, verde para quemarla". Ya'xholenil es "verdor", mientras que ya'xhule'n se refiere al "color del cielo" y ya'xk'ole'n al "aclarado tiempo, despejando nubes". Ya'xila' es "agua muy honda sin suelo", mientras que para u ya'axil $h a$ ' se dice que es "el color verde que toma el agua por profundidad". Ya'ax ka'an es "arco celeste [arco iris]".

En el diccionario de Bolles yax-kax es "a certain soil between red and black"; yaxil tun es "piedra preciosa” (probablemente jade).

Fray Thomás de Coto indica en su Thesaurus los siguientes significados de la palabra rax: "azul color, color [verde], fresca cosa, húmedo, muy". Rax camas es "caerse muerto", rax hal: qa "mazorca”, rax humuhuh: chi significa "relumbrar"; mientras que rax mae: $t i$, "enfriarse". Rax maqamoh es "color [morado]" y "rax ninoh' es "azul color [de cielo]". Rax vach es "miel”. Rax vinak significa "carne, salud, sana cosa"; rax vinakil "color, libertad"; mientras que rax vinakir: qui, "resucitar". Rax $3 a b$ es "rocío" y raxqore: qui es "admirable cosa". Rax qoroqoh se refiere a "azul color, sereno estar" y rax qoroqoh chic ah al "tiempo sereno". Raxal: nima es "estado de gloria”. Raxar: ti se refiere a "color" y a "húmedo". Raxon significa “azul color, joyas, pájaro", mientras que raxon: un 3u3, un es "doncella”.

Aparte de estos significados, fray Francisco Ximénez señala los siguientes para rax: "cosa verde o azul; fruta sin madurar; carne no cocida; muerte temprana o súbita; riquezas, gloria; cosa húmeda; manta verde". Rax uinak denota al "hombre sano", raxa3an a "cosa hermosa”. Raxporopoh significa "tiempo de bonanza", rax- 
teu designa a la "calentura con frío", mientras que raxtihe "enfriarse". Raxtunah, tin designa "embijar el cuerpo con colores". Raxuachir, ti significa "ponerse algo en efecto".

\section{Colores derivados}

En cuanto a esta categoría de términos cromáticos, los diccionarios revelan la existencia de colores percibidos como mezclas de los básicos para referir un espectro más amplio. Tal es el caso al combinar chak, ek', k'an, sak y ya'ax con otras palabras.

Los sustantivos compuestos con chak se refieren a castaño, bermejo o enrojecer, así como a encarnado oscuro; también designa a lo candente, al color de fuego ígneo; es rubio o rubio encendido, mientras que en otras composiciones se refiere al color entre colorado y azul, morado y bermejo.

En torno a las palabras compuestas con $e k^{\prime}$, se encuentran los siguientes significados: "color castaño", "negrura”, "gris", "ceniciento", "asoleado" y "tostado", así como "adquirir color ceniciento" y "bajar el color negro".

Por lo que incumbe a k'an y sus derivados, hay cuatro entradas en el Cordemex. K'an k'an se refiere a "pardo y blanco", mientras que k'anba sak significa "parda cosa de color ceniza". Es interesante señalar que los mayas podían designar blanco y negro con una sola palabra (reduplicada). Como previamente apunté, aparte de significar amarillo, la palabra k'an cubre también el espectro de rojo, encarnado y bermejo.

La palabra hoybesah posee como elemento constitutivo sah, el cual es lo mismo que sak, y significa "blanquear pared con agua de cal, matizar". De esta acepción se puede inferir que el blanco se añadía a las mezclas de colores para obtener diferentes matices. Sas ek' es "color pardo, un poco negro, entre moreno y blanco". La combinación de blanco y negro también la vimos en el caso del color k'an.

Es interesante notar que el arcoíris es ya'ax ka'an en el Cordemex, mientras que en el Diccionario k'iche de Christenson es räxkiaq'ab, donde räx es "verde/azul" y kiaq es "rojo".

\section{Consideraciones finales}

Cada lengua organiza su vocabulario conforme a su propia vivencia cultural, es decir, según las necesidades y hábitos particulares con significación e importancia para sus hablantes. Tal es el caso de las palabras y las expresiones de las lenguas mayas que pertenecen al campo semántico de los colores, organizados en un sistema taxonómico de una manera propia y particular. Según Berlin y Kay, tzeltal y tzotzil pertenecen al estado IV por reconocer cinco términos de colores 
básicos. Por otro lado, vimos que los mayas reconocen muchos colores del espectro visual, pero los cinco forman parte importante de la ideología que abarca todos los niveles de la vida, tanto el sistema religioso como múltiples aspectos de la vida cotidiana.

Con base en la información obtenida al revisar los significados de los colores en distintos diccionarios de las lenguas mayances, se nota que un mismo término puede conllevar distintos sentidos que en la gran mayoría de los casos dependen de los referentes con los que normalmente se combinan las palabras; además, estas son altamente polisémicas, haciendo que la comprensión de sus significados se dificulte aún más.

Los aspectos típicos con los que se asocia el blanco es con la claridad, limpieza, resplandor, suavidad, el alba y lo no cocido. Como color, sus referentes más comunes son las nubes y la espuma, característicos para todas las regiones donde se hablan los idiomas mayances, mientras que para las lenguas de los altos el blanco se asocia también con la nieve y el granizo, formando parte éstas dos últimas nociones de las vivencias particulares de algunos grupos. El negro es su contrario: se relaciona con la oscuridad, la noche, la suciedad, lo espantoso y lo demasiado cocido. Por consiguiente, a partir de la oposición de estos dos colores, podemos hablar de dualidades como día y noche, presencia y ausencia de luz y, posiblemente, vida y muerte.

Por ejemplo, el negro es el color de la tristeza, puesto que se refiere al luto e indica la muerte. Sin embargo, no queda claro si la costumbre de vestirse de negro en caso de muerte se emplea a partir de la llegada de los españoles o igualmente podría haber sido un hábito prehispánico. El blanco designa las canas, es decir, denota las personas ya viejas y, por lo tanto, indica la cercanía de la muerte, pero también se refiere a la sabiduría acumulada por los años de vida de estas personas. En cuanto al estado de la salud, sak, k'an y ya'ax denotan a las personas pálidas, descoloridas o blanquizas, todo ello provocado por algún malestar. En este sentido se dice del blanco que se aproxima a la muerte y, quizás, también las acepciones de otros dos colores por perder las personas su vigor vital. Por otra parte, como si fuera color por excelencia, el significado del rojo se inclina hacia "dar color a lo descolorido". Quizás en este sentido sak, k'an y ya'ax sea la oposición a chak y ek', como relación sin color-con color, es decir, no saturado-saturado.

Respecto a la temperatura, la oposición blanco-negro se manifiesta como insuficiencia-exageración. En este aspecto, el rojo es el color que antes que cualquier otro se refiere a la noción de la temperatura y representa el justo medio entre el blanco y el negro. Además, se nota que también el amarillo abarca la acepción del calor, mientras que ya'ax la del frío. De aquí se podría inferir que los colores se pensaron también en categoría de caliente-frío (rojo, amarillo-blanco, negro, $\left.y a^{\prime} a x\right)$.

Con relación a la palabra chakan, "plumas de la cola de guacamaya", en el Popol Vuh se narra cómo a los héroes gemelos se les somete a una serie de 
pruebas estando en Xibalbá, siendo una de ellas el pasar toda la noche en la Casa Oscura, con el ocote prendido sin que se consumiera. En vez de usar la raja de ocote, "pusieron una cosa roja en su lugar, o sea unas plumas de la cola de la guacamaya, que a los veladores les pareció que era ocote encendido" (Popol Vuh, 2003: 82-83. El subrayado es mío). Se identifica así al fuego con las plumas rojas de guacamaya.

Al hablar de los colores blanco y rojo, Bolles cita determinadas partes de la traducción del Ritual de los Bacabes y de Ethno-Botany de Ralph L. Roys, así como A Comparative Study of the Mayas and the Lacandons de Alfred M. Tozzer:

Sac-nicte ("white nicte"), Plumeria alba L., prescribed for throat complaints, aching bones, convulsions, and as a charm for flatulence. The chac-nicte ("red nicte") and the sac-nicte were considered to be the father and mother of the head of the Lacandón pantheon. The sac-nicte is cited in an incantation for erotic-seizure (Bolles, 2004: 14 (apartado $Z$ ]).

Esto me parece interesante porque precisamente en el Ritual de los Bacabes, traducido por Ramón Arzápalo Marín, en el "Texto VI para curar el trance erótico" los colores blanco y rojo se refieren a los principios masculino y femenino, respectivamente. Se menciona la unión de la sustancia roja con la sustancia blanca, que es uno de los casos de uso metafórico para los miembros masculino y femenino, es decir, el semen y el sangrado de la mujer:

Firmemente asentados estaban

La sustancia roja

Y la sustancia blanca.

Y ocurrió el nacimiento

Del miembro masculino

Y del miembro femenino (Ritual de los Bacabes, 1987: 289).

Igualmente, la relación blanco-masculino y rojo-femenino se encuentra en el Popol Vuh. En el tercer capítulo del libro se relata la historia de la muchacha Ixquic, ${ }^{17}$ "La de la sangre", que era la hija del dios de la muerte y la futura madre de la segunda pareja de héroes gemelos Hunahpú e Ixbalanqué. Al visitar el jícaro, la calavera de Hun-Hunahpú le habló, pidiéndole extender su mano derecha en la que arrojó su saliva, preñándola así. Es probable que su palma represente el órgano reproductor femenino y su nombre se asocie al rojo, es decir, con el sangrado femenino, mientras que la saliva, como elemento blanco, quizá se refiera al semen masculino.

El amarillo apunta a la naturaleza en su sazón y en decadencia. Por otro lado, es el color del sol, de la energía diurna. Verde y azul no se separan en la taxo-

\footnotetext{
${ }^{17}$ La palabra quic en quiché significa "sangre". Es precedida por el prefijo ix, el cual se refiere a lo femenino.
} 
nomía maya. Ya'ax apunta a lo tierno, húmedo, joven y fresco; por consiguiente, indica el origen y el principio, además de significar la naturaleza en crecimiento, por lo que se opone al amarillo. Como azul, ya'ax se asocia con la claridad celeste; como verde se refiere al tiempo de aguas y, por ello, a la abundancia vegetal y la vida terrenal en general. Ya'ax también es el color de lo profundo de las aguas. Posiblemente el azul y el verde indican la relación arriba-abajo, como constatamos anteriormente en el relato del Popol Vuh sobre las deidades creadoras. Además, la relación entre k'an y ya'ax se puede pensar como oposición de dos grandes divisiones del año solar que son las principales características climáticas del subtrópico: la temporada de secas y la de lluvias. Cuando se trata de los estados emocionales, ya'ax toma como referencia principal a la naturaleza para hablar de la serenidad. Así, se hace referencia al color del cielo y al tiempo aclarado, tiempo de bonanza cuando no hay tormentas.

Al mismo tiempo, k'an y ya'ax unen sus particulares nociones cuando se refieren a lo precioso, a la gloria, a las riquezas, pensando, antes que nada, en las piedras preciosas, la miel y las plumas del pájaro raxon (las cuales fueron usadas por los altos dignatarios). En este sentido, ya'ax también se refiere a la cosa admirable, a la salud y la prosperidad. En varias ocasiones, en el Memorial de Sololá se habla del tributo: piedras preciosas (jade), metal, guirnaldas cosidas con plumas verdes y azules (del ave quetzal), pinturas y esculturas (Memorial de Sololá, 2006: 52), entre otras cosas.

Es interesante apuntar - siguiendo la misma fuente - el hecho de que también se tributaba tierra blanca o zahcab. Según Recinos, esta palabra tiene dos significados: "tierra blanca" o "yeso"; era una sustancia que usaban los indios para pintarse el rostro y el cuerpo (Memorial de Sololá, 2006: 53). Esto se corrobora más adelante en la narración, al describir la preparación para ir a una guerra al oriente, a Tulán: "En seguida se revistieron de sus arcos, escudos, cotas de algodón y plumas, y se pintaron con yeso” (Memorial de Sololá, 2006: 56).

Hay que notar que esta manera de pintarse el cuerpo de blanco para ir a las batallas es diferente a la descrita por Landa y en otras fuentes anteriormente presentadas, en las cuales se indica que se pintaban de negro y rojo. Con esto se señalan ciertas diferencias entre los grupos mayas que, aunque pertenecían a una cultura que compartía muchos elementos, tenían sus propios hábitos y costumbres creados en su ambiente específico y en contacto con otros grupos humanos.

Se infiere que los colores o sus combinaciones siempre se relacionan con algunos referentes del mundo social, simbolizando así el estatus de las personas. Así, k'an y ya'ax contendrían la noción "de alto rango o valor”, mientras que el blanco sería reservado para los pertenecientes a la clase social baja, porque en el mismo texto se señala que la víctima para el sacrificio se untaba con zahcab, "la tierra blanca”, antes de ser sacrificada (Memorial de Sololá, 2006: 32). ${ }^{18}$ Este

\footnotetext{
${ }^{18}$ En esta página se menciona por primera vez y se repite más adelante.
} 
hecho otra vez apunta a una costumbre diferente a la reportada en las fuentes escritas de la península de Yucatán (donde se pintaban de azul).

Sak, chak y ya'ax son intensificadores del significado de otras palabras y como tal poseen el sentido de "muy, mucho". Por otro lado, en cuanto a sak, Alfredo Barrera Vásquez acota que los significados de sak se pueden agrupar en cuatro conjuntos: 1) significando "blanco"; 2) "intensidad"; 3) "ficción, arte, artificio, artificial"; y 4) acepciones indefinidas. Es interesante en este lugar acercarse al tercer grupo; el ejemplo adecuado sería sak be (o sacbé), ya que es un camino de arte, una calzada artificial. Igualmente, aquí cabrían las acepciones de "falso" y "algo hecho a medias", como algo fingido y ficticio. Finalmente, a este autor no le sorprende el por qué de nombrar el "tejido" y "tejer" con sak, ya que se trata de algo hecho o que se hace con destreza y habilidad humana (Barrera Vásquez, 1980: 47-51).

Por último, en esta ocasión no pude nombrar todas las especies de la flora y la fauna, así como las enfermedades designadas con las palabras de un color básico. Este tema sería muy interesante para una futura investigación.

\section{BIBLIOGRAFÍA}

Arzápalo Marín, H. Ramón

1995 Calepino de Motul. Diccionario maya-español. México: Universidad Nacional Autónoma de México, Dirección General de Asuntos del Personal Académico, Instituto de Investigaciones Antropológicas.

Barrera Vásquez, Alfredo (coord.)

1980 Diccionario maya Cordemex. Maya-español. Español-maya. Redactores Juan Ramón Bastarrachea Manzano y William Brito Sansores; colaboradores Refugio Vermont Salas, David Dzul Góngora y Domingo Dzul Poot. Mérida: Cordemex.

Barrera Vásquez, Alfredo

1981 Estudios lingüísticos, Obras completas, tomo II. México: Fondo Editorial de Yucatán.

Berlin, Brent y Paul Kay

1991 Basic Color Terms, their Universality and Evolution. Berkeley/Los Ángeles/ Oxford: University of California Press.

Bolles, David

2004 Combined Dictionary-Concordance of the Yucatecan Mayan Language <http:// www.famsi.org/reports/96072/index.html > . [Consultada el 20 de mayo del 2011.]

Bourdin Rivero, Gabriel Luis

2008 "El léxico de las emociones en el maya yucateco". Tesis de doctorado. Méxi- 
co: Universidad Nacional Autónoma de México, Instituto de Investigaciones Antropológicas.

Coto, fray Thomas de

1983 [Thesavrvs verborū] Vocabulario de la lengua cakchiquel v[el] guatemalteca, nueuamente hecho y recopilado con summo estudio, trauajo y erudición, edición de R. Acuña. México: Universidad Nacional Autónoma de México, Instituto de Investigaciones Filológicas.

Christenson, Allen J.

2003 K'iche'-English Dictionary and Guide to Pronunciation of the K'iche'-Maya Alphabet, reporte entregado a Foundation for the Advancement of Mesoamerican Studies (FAMSI). < http://www.famsi.org/mayawriting/dictionary/christenson/ quidic_complete.pdf) $>$. [Consultada el 20 de mayo del 2011.]

Dehouve, Daniele

1995 "Nombrar los colores en náhuatl (siglos xvI-xx)", El color en el arte mexicano, pp. 51-95. G. Roque (coord.). México: Universidad Nacional Autónoma de México, Instituto de Investigaciones Estéticas.

Garza, Mercedes de la et al. (eds.)

1983 Relaciones histórico-geográficas de la gobernación de Yucatán: Mérida, Valladolid y Tabasco. Paleografía por María del Carmen León Cázares. México: Universidad Nacional Autónoma de México, Instituto de Investigaciones Filológicas, Centro de Estudios Mayas.

Garza, Mercedes de la y Martha Ilia Nájera Coronado (eds.)

2002 Religión Maya, Volumen 2. Madrid: Trotta (Enciclopedia Iberoamericana de Religiones, 2).

Kaufman, Terrence S. y John Justeson

2003 A Preliminary Mayan Etymological Dictionary. <http:/www.famsi.org/ reports/01051/pmed.pdf > . [Consultada el 20 de mayo del 2011.]

Kettunen, Harry y Christophe Helmke

2008 Introduction to Maya Hieroglyphs, Workshop Handbook < http://www.mesoweb. org/resourses/handbook/index.htm $>$. [Consultada el 20 de mayo del 2011.]

Landa, Fray Diego de

1985 Relaciones de las cosas de Yucatán. Edición, introducción y notas de Miguel Rivera Dorado. Madrid: Historia 16 (Crónicas de América, 7).

Libro de Chilam Balam de Chumayel

1985 Traducción de Antonio Mediz Bolio, prólogo, introducción y notas de Mercedes de la Garza. México: Dirección General de Publicaciones y Medios de la Secretaría de Educación Pública. 
Memorial de Sololá o Anales de los cakchiqueles, y Título de los señores de Totonicapán

2006 Traducción de Dionisio José Chontay, introducción de Adrián Recinos. México: Fondo de Cultura Económica.

Navarrete, Carlos

2002 Relatos mayas de Tierras Altas sobre el origen del maíz: los caminos de Paxil. México: Universidad Nacional Autónoma de México.

Popol Vuh, las antiguas historias del Quiché

2003 Traducción, introducción y notas de Adrián Recinos. México: Fondo de Cultura Económica (Colección Popular).

Ritual de los Bacabes

1987 Edición de Ramón Arzápalo Marín. México: Universidad Nacional Autónoma de México, Instituto de Investigaciones Filológicas, Centro de Estudios Mayas (Fuentes para el Estudio de la Cultura Maya, 5).

Roque, Georges (coord.)

2003 El color en el arte mexicano. México: Universidad Nacional Autónoma de México, Instituto de Investigaciones Estéticas.

Savkic, Sanja

200 "El color en el Códice Dresde según la información de las fuentes escritas y de las linguiísticas". Tesis de maestría en Estudios Mesoamericanos, México: Universidad Nacional Autónoma de México, Facultad de Filosofía y Letras.

Thompson, J. Erik S.

2004 Historia y religión de los mayas. México: Siglo XXI Editores (Colección América Nuestra).

Vargas Melgarejo, Luz María

1998 Los colores lacandones: la percepción visual de un pueblo maya. México: Instituto Nacional de Antropología e Historia (Colección Científica, Serie Antropología Física).

Ximénez, fray Francisco

1985 Primera parte del Tesoro de las lenguas cakchiquel, quiché y zutuhil, en que las dichas lenguas se traducen a la nuestra, española. Edición de Carmelo Sáenz de Santa María. Guatemala: Academia de Geografía e Historia de Guatemala (Publicación especial, 30). 
